\title{
Survival of gas phase amino acids and nucleobases in space radiation conditions
}

\author{
S. Pilling ${ }^{1}$, D. P. P. Andrade ${ }^{3}$, R. B. de Castilho ${ }^{3}$, R. L. \\ Cavasso-Filho $^{1}$, A. F. Lago ${ }^{1}$, L. H. Coutinho ${ }^{2}$, G. G. B. de Souza ${ }^{3}$, \\ H. M. Boechat-Roberty ${ }^{3}$, and A. Naves de Brito ${ }^{1}$ \\ ${ }^{1}$ LNLS, Laboratório Nacional de Luz Síncrotron, São Paulo, Brazil \\ email: sergiopilling@yahoo.com.br, cavasso@lnls.br, alago@lnls.br, \\ arndaldo@lnls.br \\ ${ }^{2}$ UEZO, Centro Universitário Estadual da Zona Oeste, Rio de Janeiro, Brazil \\ email: coutinholh@yahoo.com \\ ${ }^{3}$ UFRJ, Universidade Federal do Rio de Janeiro, Rio de Janeiro, Brazil \\ email: diana_andrade@ufrj.br, castilho@iq.ufrj.br, gerson@iq.ufrj.br, \\ heloisa@ov.ufrj.br
}

\begin{abstract}
We present experimental studies on the photoionization and photodissociation processes (photodestruction) of gaseous amino acids and nucleobases in interstellar and interplanetary radiation analogs conditions. The measurements have been undertaken at the Brazilian Synchrotron Light Laboratory (LNLS), employing vacuum ultraviolet (VUV) and soft X-ray photons. The experimental set up basically consists of a time-of-flight mass spectrometer kept under high vacuum conditions. Mass spectra were obtained using a photoelectron photoion coincidence technique. We have shown that the amino acids are effectively more destroyed (up to $70-80 \%$ ) by the stellar radiation than the nucleobases, mainly in the VUV. Since polycyclic aromatic hydrocarbons have the same survival capability and seem to be ubiquitous in the ISM, it is not unreasonable to predict that nucleobases could survive in the interstellar medium and/or in comets, even as a stable cation.
\end{abstract}

Keywords. Methods: laboratory, molecular data, astrochemistry, astrobiology

\section{Introduction}

The search for amino acids and nucleobases (and related compounds) in the interstellar medium/comets has been performed over at least the last 30 years, but unfortunately it has yet to be successful (e.g., Brown et al. 1979, Simon \& Simon 1973). However, recently some traces (upper limits) of the simplest amino acid, glycine $\left(\mathrm{NH}_{2} \mathrm{CH}_{2} \mathrm{COOH}\right.$ ), were observed in the comet Hale-Bopp (Crovisier et al. 2004) and in some molecular clouds associated with star forming regions (Kuan et al. 2003a), but these identifications have yet to be verified (Snyder et al. 2005, Cunningham et al. 2007). Despite no direct detection of nucleobases in comets or in molecular clouds, some of their precursor molecules like $\mathrm{HCN}$, pyridines, pyrimidines and imidazole were been reported in the Vega 1 flyby of comet Halley (Kissel \& Krueger, 1987) and have been searched for in the interstellar medium (Kuan et al. 2003b).

The search for these biomolecules in meteorites, on the contrary, has revealed an amazing number of proteinaceous and non-proteinaceous amino acids, up to 3 parts per million (ppm) (e.g., Cronin 1998 and references therein), and some purine and pyrimidine based nucleobases up to 1.3 ppm (e.g., Stocks \& Schwartz 1981 and references therein). This dichotomy between the carbonaceous chondrites meteorites and interstellar medium/comets remains a big puzzle in the field of astrochemistry and in investigations about the origin of life. 
The goal of this work is to review some experimental gas-phase photoionization and photodissociation studies of amino acids and nucleobases induced by vacuum ultraviolet (VUV) and soft X-ray photons, obtained recently by our group (Lago et al. 2004, Coutinho et al. 2005, Marinho et al. 2006, Pilling et al. 2007b). A possible direction on the different survivability of these biomolecules on astrophysical environments are given.

\section{Experimental methodology and results}

In an attempt to simulate the stellar/solar VUV and soft X-ray flux we have used synchrotron radiation as a light source. The experiments were performed at the Brazilian Synchrotron Light Laboratory (LNLS), employing harmonic free VUV photons (CavassoFilho et al. 2007) and soft X-ray photons from the toroidal grating monochromator (TGM) beamline. The emergent photon beam flux was recorded by a light sensitive diode. Briefly, the radiation $\left(\sim 10^{12}\right.$ photons $\left.\mathrm{s}^{-1}\right)$ from the beamline perpendicularly intersect the vapor-phase sample at the center of the ionization region inside a high vacuum chamber (Boechat-Roberty et al. 2005, Pilling et al. 2006). Mass spectra were obtained using the photoelectron photoion coincidence (PEPICO) technique (Pilling et al. 2007b, c and references therein).

In Figure 1 we have shown the time-of-flight mass spectra of the fragments released from the amino acid glycine and the nucleobase adenine, recorded at different photon energies over the VUV $(12-21 \mathrm{eV})$ and soft X-ray $(\sim 150 \mathrm{eV})$ ranges. As a general rule, even at low photon energy, the amino acids have only a small contribution $(\lesssim 10 \%)$ or even were not detected, a consequence of their high photodestruction degree. The release of the carboxyl group $(\mathrm{COOH})$ as a neutral or cationic species, depending on the amino acid, is one of the most import dissociation channels (see also Jochims et al. 2004). The nucleobases have shown a higher molecular stability in comparison with the amino acids. For these molecules, the parent ions remain relatively strong over all the studied VUV photon energy range. In all spectra, as the photon energy increases, the fragmentation profile also increases, as expected. Some minor contamination of water was observed in the spectra, which reflects the high hydrophilic character of the samples.

In previous studies of photodissociation of nucleobases (e.g., Jochims et al. 2005, Schwell et al. 2006) in the VUV photon energy range the authors have identified some important photodissociation channels as well as HNCO loss from thymine and uracil and HCN loss from adenine. As pointed out by Pilling et al. (2007b), in the case of

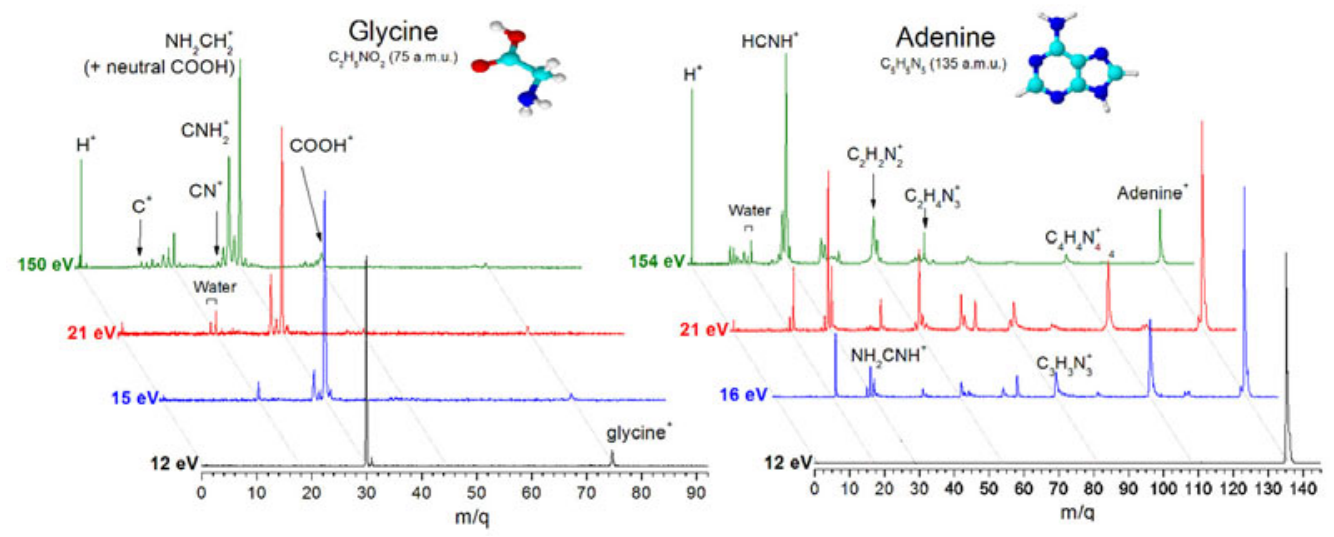

Figure 1. Time-of-flight mass spectra of gaseous amino acid glycine and nucleobase adenine recorded at different photon energies at VUV $(12-21 \mathrm{eV})$ and at soft X-ray $(\sim 150 \mathrm{eV})$ ranges. 


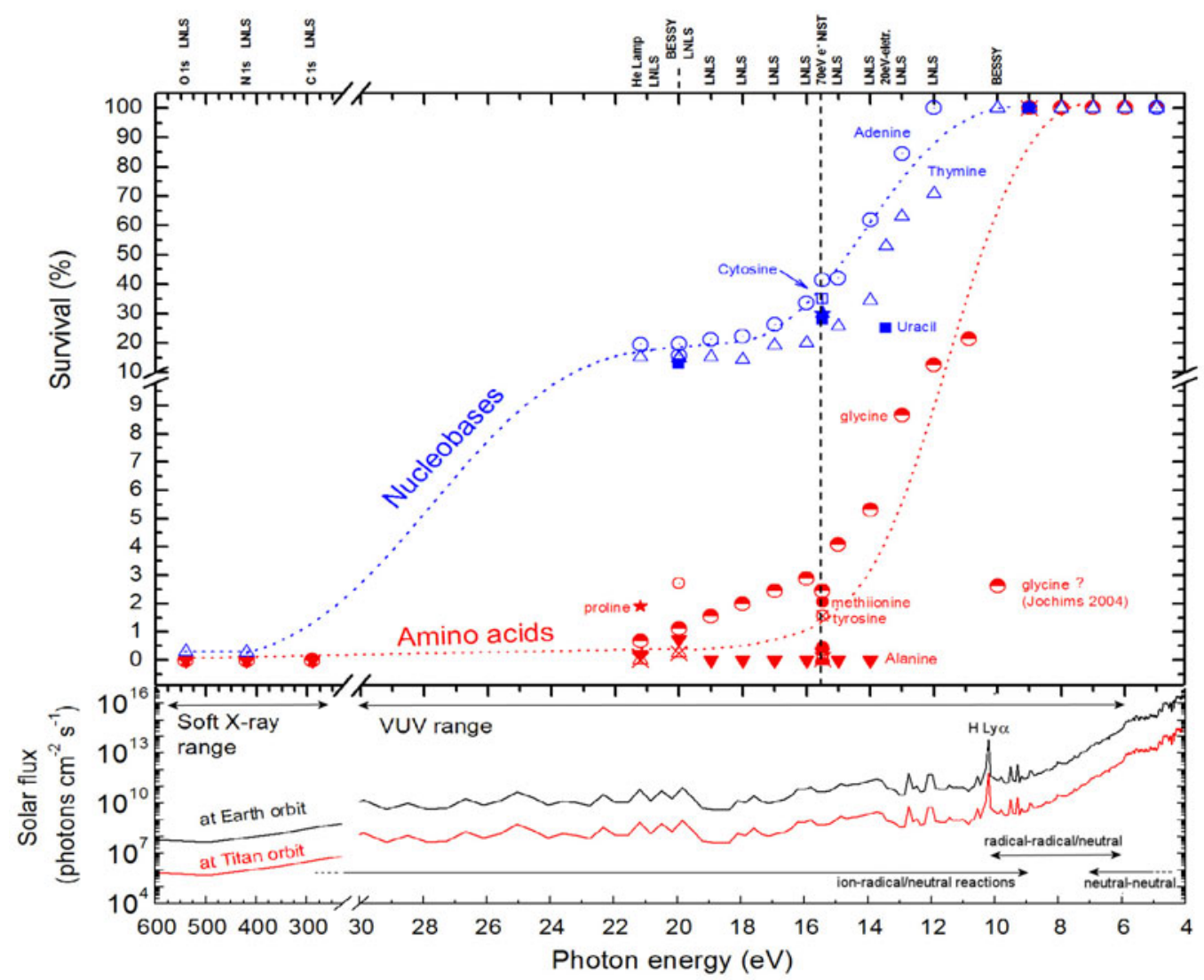

Figure 2. (Top) Comparison between the survival (photoresistence) of different amino acids and nucleobases due to ionizing radiation field in the VUV and soft X-ray. (Bottom) The solar photon flux at Earth and Titan orbit (adapted from Gueymard 2004). See details in text.

adenine, the neutral HCN may represent as much as $40 \%$ of its photodissociative channels. The ion $\mathrm{HCNH}^{+}$is another largely photoproduced fragment from both amino acids and nucleobases.

A comparison between the survival of different nucleobases and amino acids due to ionizing radiation field from 4 to $600 \mathrm{eV}$ is given at Figure 2. Our data is represented by LNLS labels. The literature data were gather mainly from the NIST database and Jochims et al. $(2004,2005)$. The amino acids are effectively more destroyed by VUV stellar/solar radiation than the nucleobases. The higher resistance of nucleobases to the ionizing photons may be associated with the presence of the hetero-cyclic structure and unsaturated bonds.

For comparison, we also present in the bottom panel of Figure 2, the solar photon flux at ultraviolet and soft X-rays at Earth and Titan orbit (adapted from Gueymard 2004). The different photochemical domains are also given. According to the detailed review of Schwell et al. (2006), the photoabsorption cross section of these molecules is higher in VUV as compared to the mid-UV $(<6 \mathrm{eV})$. In particular, all molecules absorb strongly at $10.2 \mathrm{eV}$, where the intense $\operatorname{Ly} \alpha(10.2 \mathrm{eV})$ stellar/solar emission is located. Most of the small biomolecules studied have first ionization energies (IE) below this energy, making photoionization phenomena an important issue to study. Since most of the amino acids has their first AE below $10.2 \mathrm{eV}$, the stellar/solar hydrogen Ly $\alpha$ has a great influence 
on their molecular survival. This is not observed in the case of nucleobases, which have their first AE at energies above the hydrogen Ly $\alpha$ energy, where the photon flux is about 2 orders of magnitude lower (in the case of Sun).

\section{Conclusion}

We have shown that the amino acids are effectively more destroyed by stellar radiation than the nucleobases, mainly in the VUV spectral range where the differences reach up 70-80\%, decreasing to high-energy photon range, corroborating other experimental results given in the literature. The nucleobases are able to form a stable cation in the gas phase and since polycyclic aromatic hydrocarbons (PAHs) and polycyclic aromatic nitrogen-rich hydrocarbons (PANHs) have the same capability and seem to be ubiquitous in the ISM (Allamandola et al. 1986), it is not unreasonable to predict that aromatic nucleic acid bases could survive in the interplanetary and interstellar media.

These results lead us to ask an interesting question. Why don't we find nucleobases in cometary/molecular clouds radioastronomical observations since they are more resistant to stellar ultraviolet radiation than the detected amino acid (e.g., glycine)? Probably, the answer may be associated with the formation pathway efficiencies rather than with the detection limits and more studies of this subject are need.

Finally, a possible direction to the search of large pre-biotic molecules, as the case of amino acids and nucleobases or even for larger molecules, could be the search not for the molecules themselves, but from their photoproduced daughter species like, for example, the fragments $\mathrm{COOH}^{+}, \mathrm{HNCO}^{+}, \mathrm{HCN}^{+}$, and $\mathrm{HCNH}^{+}$. The abundances, derived from the radio-observation of these fragments, combined with the laboratory data (e.g., relative ion yield and photoproduction cross section) may give us a clue about the presence and the amount (upper limit) of their parent molecules.

\section{References}

Allamandola, L. J., Tielens, A. G. G. M., \& Barrer, J. R. 1986, ApJS, 71, 733.

Boechat-Roberty, H. M., Pilling, S., \& Santos, A. C. F. 2005, A\&A, 438, 915.

Brown, R. D., Godfrey, P. D., Storey, J. W. V., Bassez, M.-P., et al. 1979, MNRAS, 186, 5P.

Cavasso-Filho, R. L., Homem, M. G. P., Lago, A., Pilling, S., \& Naves de Brito, A. 2007, J El. Spect. Rel. Phen., 156-158, 168.

Coutinho, L. H., Homem, M. G. P., Cavasso-Filho, R. L., Marinho, R. R. T., Lago, A.F., de Souza, G. G. B., \& Naves de Brito, A. 2005, Braz. J. Phys., 35, 940.

Cronin, J. R. 1998, in: A. Brack (eds.), The Molecular Origins of Life, (Cambridge University Press, UK)

Crovisier, J., Bockelée-Morvan, D., Colom, P., Biver, N., Despois, D., Lis, D.C., et al. 2004, $A \mathscr{E} A, 418,1141$.

Cunningham, M. R., Jones, P. A., Godfrey, P. D., et al. 2007, MNRAS, 376, 1201.

Gueymard, C. A. 2004, Solar Energy, 76, 423.

Jochims, H.-W., Schwell, M., Baumgartel, H., \& Leach, S. 2005, Chem. Phys., 314, 263.

Jochims, H.-W., Schwell, M., Chotin, J-.L., Clemino, M., Dulieu, F., Baumgärtel, H., \& Leach, S. 2004, Chem. Phys., 298, 279.

Kissel, J. \& Krueger, F. R. 1987, Nature, 326, 755.

Kuan, Y.-J., Charnley, S. B., Huang, H.-C., Tseng, W.-L., \& Kisiel, Z. 2003a, ApJ, 593, 848.

Kuan, Y.-J., Yan, C.-H., Charnley, S. B., Kisiel, Z., Ehrenfreund, P., \& Huang, H.-C. 2003b, $M N R A S, 345,650$.

Lago, A. F., Coutinho, L. H., Marinho, R. R. T., Naves de Brito, A., \& de Souza, G.G.B. 2004, Chem. Phys., 307, 9.

Marinho, R. R. T., Lago, A. F., Homem, M. G. P., Coutinho, L. H., de Souza, G. G. B., \& Naves de Brito, A. 2006, Chem. Phys., 324, 420.

Pilling, S., Andrade, D. P. P., Neves, R., Ferreira-Rodrigues, A. M., Santos, A.C.F., \& BoechatRoberty, H. M. 2007a, MNRAS, 375, 1488. 
Pilling, S., Lago A. F., Coutinho, L. H., de Castilho R.B., de Souza, G. G. B., \& Naves de Brito, A. 2007b, Rapid Commun. on Mass Spectrom., 21, 3646.

Pilling, S., Neves, R., Santos, A. C. F., \& Boechat-Roberty, H. M. 2007c, A\&SA, 464, 393.

Pilling, S., Santos, A. C. F., \& Boechat-Roberty, H. M. 2006, A\&A, 449, 1289.

Schwell, M., Jochims, H.-W., Baumgärtel, H., Dulieu, F., \& Leach, S. 2006, PESSS, 54, 1073.

Simon, M. N., \& Simon, M. 1973, ApJ, 184, 757.

Snyder, L. E., Lovas, F. J., Hollis, J. M., Friedel, D. N., Jewell, P. R., Remijan, A., Ilyushin, V. V., Alekseev, E. A. \& Dyubko, S. F. 2005, ApJ, 619, 914.

Stocks, P. G. \& Schartz, A. W. 1981, Geochim Cosmochim. Acta, 45, 563.

\section{Discussion}

KoBAYASHI: You irradiate with UV and X-rays. How many photons did you expose these molecules to, and how does this correspond to exposure times in space?

PILling: Our flux is about $10^{12}$ photons per second in the ultraviolet. At the moment, I don't know how this corresponds to exposure time in the interstellar environment.

ZiURYs: Some of those products you proposed from your fragmentation are seen in the interstellar medium, for example, $\mathrm{COOH}^{+}$and $\mathrm{HCNH}^{+}$. We have no information on some of the other molecules because we don't know their rest frequencies.

PILling: We are proposing that if you combine the radio observations for molecules like $\mathrm{COOH}^{+}$, together with the branching ratios from the amino acids from the lab, maybe we can trace some upper limits from these molecules.

ZIURYS: Yes, look at the relative abundances and look at the branching ratios. It's a good idea. 


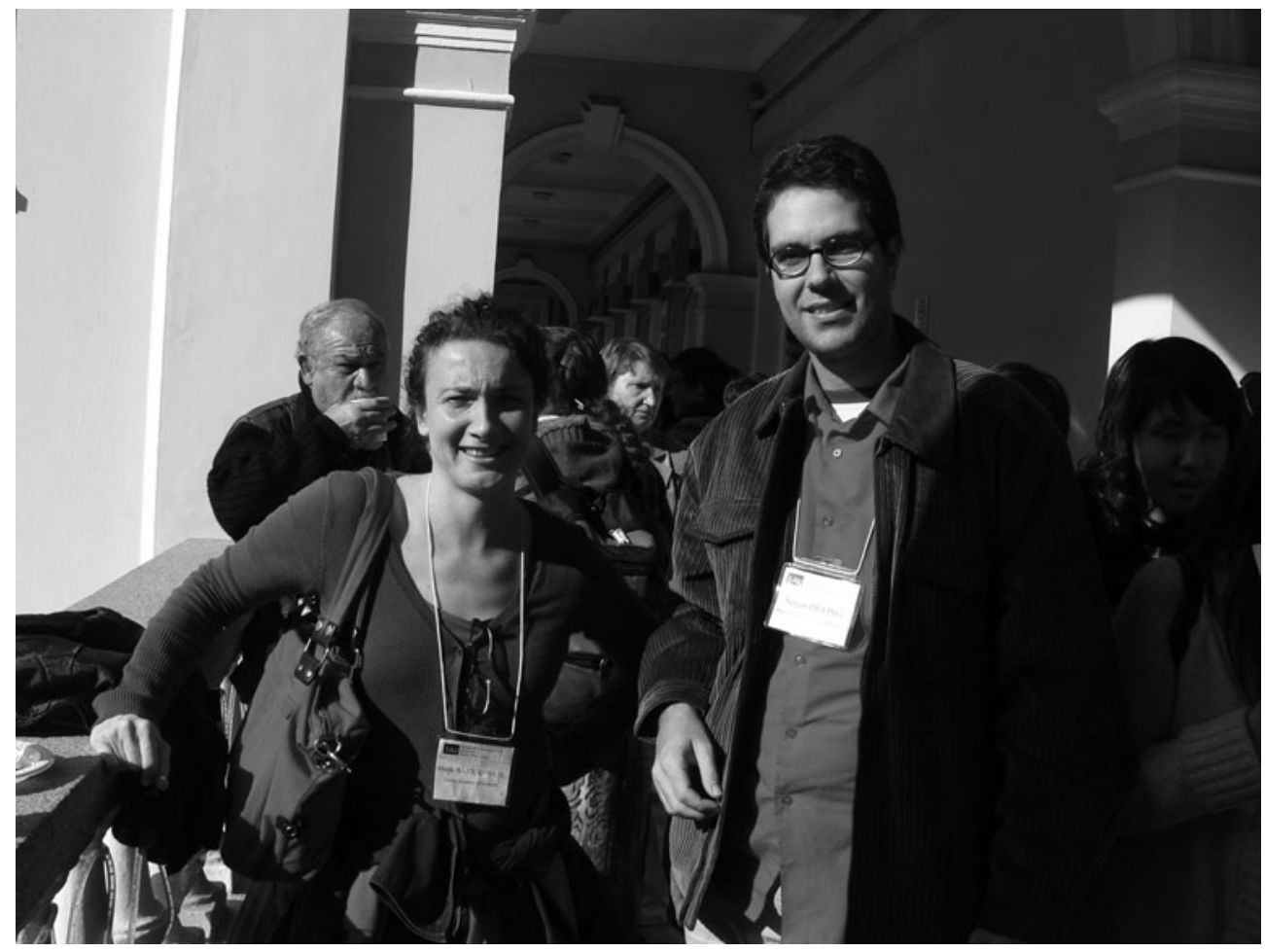

Mia Hajdukova and Sergio Pilling (photo by Mia Hajdukova). 